\title{
Minimally Invasive Transforaminal Lumbar Interbody Fusion: Comparison of Grade I Versus Grade II Isthmic Spondylolisthesis
}

DUSTIN H. MASSEL, BS, BENJAMIN C. MAYO, BA, WILLIAM W. LONG, BA, KRISHNA D. MODI, BS, GREGORY D. LOPEZ, MD, GRANT D. SHIFFLETT, MD, BRYCE A. BASQUES, MD, PHILIP K. LOUIE, MD, DANIEL D. BOHL, MD, MPH, FADY Y. HIJJ, BS, ANKUR S. NARAIN, BA, KERN SINGH, MD Department of Orthopaedic Surgery, Rush University Medical Center, 1611 W. Harrison St. Suite \#300, Chicago, Illinois

\begin{abstract}
Background: Minimally invasive transforaminal lumbar interbody fusion (MIS-TLIF) is often used to treat lowgrade isthmic spondylolisthesis (IS). No studies have compared surgical outcomes for grade I and II IS following MISTLIF. Therefore, the objective of the current study was to compare outcomes between patients with grade I and II IS following MIS-TLIF.

Methods: A retrospective cohort analysis was performed on a prospectively maintained database of patients who underwent a primary 1-level MIS-TLIF for treatment of IS between 2007 and 2015. Grade I patients underwent a unilateral tubular approach with a single interbody cage and bilateral pedicle screw instrumentation. Grade II patients underwent a bilateral tubular approach with bilateral interbody cage and pedicle screw placement. Baseline patient demographics and characteristics were compared using Student $t$ test and $\chi^{2}$ analysis. Differences in peri- and postoperative outcomes were assessed using Poisson regression with robust error variance or linear regression adjusted for perioperative variables.

Results: A total of 58 patients with IS underwent MIS-TLIF; 21 (36.2\%) were grade I and 37 (63.8\%) were grade II. The grade I cohort was younger (42.2 versus 50.6 years, $P=.029$ ); no other differences in preoperative variables were observed. No significant differences in operative time, estimated blood loss, length of hospital stay, postoperative visual analogue scale scores, or complication and revision rates were demonstrated between cohorts. Arthrodesis rate was lower in the grade I cohort, though not statistically significant.

Conclusions: Despite the grade I cohort being younger with less-severe diagnoses, the grade II cohort experienced similar outcomes. This finding may be due to the grade II cohort receiving bilateral cages, potentially providing a better fusion environment.

Clinical Relevance: These results suggest that MIS-TLIF provides sufficient stabilization and fusion for treatment of grade II IS despite increased vertebral body displacement. In addition, MIS-TLIF with bilateral approach and interbody cage placement should be examined for treatment of high-grade IS cases.
\end{abstract}

\section{Research Article}

Keywords: minimally invasive spine surgery, transforaminal lumbar interbody fusion, low-grade, isthmic spondylolisthesis, bilateral/unilateral interbody cage

\section{INTRODUCTION}

Minimally invasive (MIS) and open transforaminal lumbar interbody fusions (TLIF) are accepted surgical procedures for the definitive treatment of common spinal pathologies, including isthmic spondylolisthesis (IS). ${ }^{1-3}$ However, due to the severity of approach-related and postoperative morbidity, the surgical paradigm is shifting toward a more minimally invasive approach. MIS-TLIF results in smaller incisions, limited soft tissue injury, reduced estimated blood loss (EBL), decreased postoperative pain, more rapid improvement in patient function, and shorter length of hospital stay (LOS) compared with the open procedure. ${ }^{4-6}$

IS is the anterior subluxation of the cranial over the caudal vertebra caused by a defect in the pars interarticularis or spondylolysis, affecting $5 \%$ to $8 \%$ of the population. ${ }^{7-11}$ Commonly asymptomatic, IS is often incidentally found in children and adolescents as the result of progression of bilateral defects in the pars interarticularis. Most cases of spondylolysis and IS occur at the L5 and L5-S1 levels, respectively. ${ }^{12}$ IS is hypothesized to result from 
stress fractures that develop secondary to repetitive trauma as seen in sports that require loading the lumbar spine in hyperextension such as gymnastics, football, and volleyball. For the majority of patients, removal of the offending activity with a course of dedicated physical therapy results in resolution of symptoms. Adolescent patients may remain asymptomatic for many years. However, long standing intersegmental instability can lead to further anterolisthesis due to degenerative changes in the intervertebral discs during the fourth and fifth decades of life. Progression of the spondylolisthesis may result in symptomatic mechanical low back or radicular leg pain., $8,11,13$

Indications for surgical management of IS include failed conservative management for greater than 6 months, progressive symptomatic low-grade IS, or any high-grade IS in order to prevent neurologic dysfunction, additional deformity, and pain. $^{7,11,13,14}$ However, due to the potentially complicated anatomy and technical difficultly associated with the complex three-dimensional deformity, the treatment options for high-grade IS remain controversial. ${ }^{8}$

Common procedures performed to treat IS include decompression, in situ or instrumented fusion and reduction, anterior (ALIF), lateral (LLIF), posterior (PLIF), and transforaminal (TLIF) lumbar interbody fusion. ${ }^{15}$ The TLIF procedure is often used to avoid the morbidity and surgical complexity associated with an anterior approach. Furthermore, the iliac crest precludes the ability to access the L5-S1 space via a lateral approach. To date, however, there is limited literature regarding the use of MIS TLIF for the treatment of IS. The purpose of this study is to compare the outcomes of MIS TLIF for the treatment of grade I versus grade II IS.

\section{MATERIALS AND METHODS}

\section{Patient Population}

Following institutional board review approval (ORA\# 14051301), a prospectively maintained surgical database of patients was retrospectively reviewed. Patients who underwent a primary singlelevel MIS-TLIF for the treatment of IS between 2007 and 2015 were identified. All patients with less than 6 months of postoperative follow-up, grade III IS and above, and those with both IS and degenerative spondylolisthesis (DS) were excluded.

\section{Demographic and Outcome Analysis}

Patients were divided in to grade I and grade II (Figures 1A, 1B) cohorts based on their radiographically diagnosed IS Meyerding grade. ${ }^{16}$ Patients were characterized in terms of the following demographic, comorbidity, and operative factors: age, gender, body mass index $(<25,25-29,30-35$, $\left.\geq 35 \mathrm{~kg} / \mathrm{m}^{2}\right)$, smoking status, primary insurance (Medicare, Workers' Compensation, or Commercial Payer), and Charlson Comorbidity Index (CCI). Operative factors analyzed included operative level (L4-L5, L5-S1), duration of procedure, EBL, LOS, and pre- and postoperative visual analogue scale (VAS) pain scores. Arthrodesis was determined by computed tomography (CT) scan evaluation of the fusion mass and demonstration of bony bridging on three sequential cuts in the sagittal and coronal planes, as well as no evidence of subchondral cysts, end plate sclerosis, or haloing around the interbody cage(s) or pedicle screws.

\section{Surgical Technique}

Grade I patients underwent a unilateral tubular approach, whereas grade II patients underwent a bilateral tubular approach. The paraspinal skin incisions were made using the Wiltse technique under fluoroscopy. Bilateral pedicle screws were placed percutaneously over guide wires. A unilateral laminotomy, facetectomy, decompression, and placement of a single interbody cage was performed through a $21-\mathrm{mm}$ nonexpandable tubular retractor for the grade I cohort. In the grade II cohort, this procedure was repeated on the contralateral side to allow for placement of an additional interbody cage. Prior to TLIF cage insertion, local bone graft that had been collected from the laminectomy and facetectomy was morselized and mixed with $5 \mathrm{cc}$ of bone marrow aspirate from the cannulated pedicles and $15 \mathrm{cc}$ of allograft cancellous bone. This mixture was impacted to the front of the disk space. Each TLIF cage was prepared with the same mixture placed within the interbody cage with the addition of an extra small kit rhBMP-2 $(2.1 \mathrm{mg})$ or a small kit rhBMP-2 (4.2 $\mathrm{mg})$ for the single or bilateral interbody cages, respectively. A single interbody cage was used for grade I IS cohort and bilateral interbody cages (Figures 1C, 1D) for the grade II IS cohort. No posterolateral fusion was performed. Midline muscular and ligamentous structures were all preserved during the procedure. 


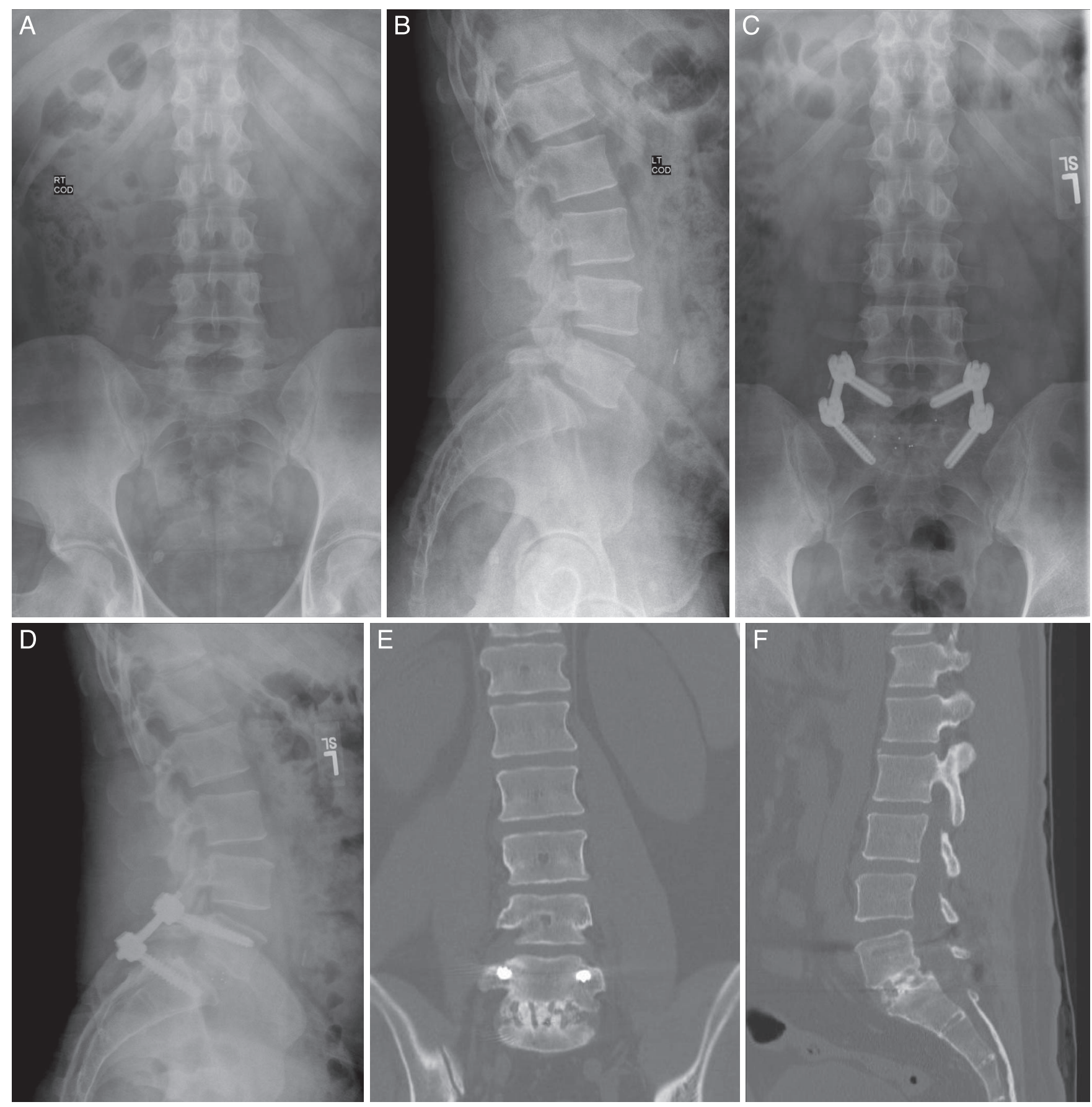

Figure 1. (A, B) Preoperative AP and lateral radiographs of a 42-year-old man with Meyerding grade II isthmic spondylolisthesis at L5-S1. (C, D) Postoperative AP and lateral radiographs of the patient following MIS TLIF with bilateral approach and bilateral interbody cage placement. (E, F) Postoperative coronal and sagittal computed tomography (CT) scan of the patient demonstrating arthrodesis at 1-year.

\section{Statistical Methods}

Statistical analysis was conducted using Stata/ MP 13.1 for Mac (StataCorp LP, College Station, Texas). Differences in patient demographics, comorbidities, and procedural characteristics were analyzed using Pearson $\chi^{2}$ and independent sample $t$ test for categorical (sex, body mass index, smoking status, insurance payer status) and continuous (age, comorbidity burden [CCI], preoperative VAS) data, respectively. Multivariate linear and Poisson regression with robust error variance was used to test for procedural characteristics (operative time, EBL, LOS, postoperative VAS, change in VAS) and postoperative outcomes (complications, pseudarthrosis, and arthrodesis rates) adjusted for all previously mentioned demographic, comorbidity, 
Table 1. Baseline characteristics. ${ }^{a}$

\begin{tabular}{|c|c|c|c|}
\hline & \multicolumn{2}{|c|}{ Isthmic Spondylolisthesis $^{\mathrm{b}}$} & \multirow[b]{2}{*}{$P$ Value } \\
\hline & Grade I $(N=21)$ & Grade II (N = 37) & \\
\hline Age,mean $\pm \mathrm{SD}, \mathrm{y}$ & $42.2 \pm 14.7$ & $50.6 \pm 13.1$ & .029 \\
\hline Sex, \% (n) & & & .747 \\
\hline Female & $47.6(10)$ & $43.2(16)$ & \\
\hline Male & $52.4(11)$ & $56.8(21)$ & \\
\hline Body mass index, \% (n) & & & .331 \\
\hline Nonobese (BMI < 30) & $38.1(8)$ & $51.4(19)$ & \\
\hline Obese $($ BMI $\geq 30)$ & $61.9(13)$ & $48.7(18)$ & \\
\hline Smoking status, \% (n) & & & .328 \\
\hline Nonsmoker & $66.7(14)$ & $78.4(29)$ & \\
\hline Smoker & $33.3(7)$ & $21.6(8)$ & \\
\hline Level of spondylolisthesis, \% (n) & & & .760 \\
\hline L4-L5 & $28.6(6)$ & $32.4(12)$ & \\
\hline L5-S1 & $71.4(15)$ & $67.6(25)$ & \\
\hline Insurance, \% (n) & & & .430 \\
\hline Medicare & $14.3(3)$ & $10.8(4)$ & \\
\hline Worker's compensation & $57.1(12)$ & $43.2(16)$ & \\
\hline Commercial & $28.6(6)$ & $46.0(17)$ & \\
\hline Comorbidity burden (CCI) & $2.1 \pm 2.4$ & $2.4 \pm 1.8$ & .551 \\
\hline Preoperative VAS, mean $\pm \mathrm{SD}$, min & $7.3 \pm 1.8$ & $7.3 \pm 1.7$ & .986 \\
\hline
\end{tabular}

Abbreviations: SD, standard deviation; CCI, Charlson comorbidity index; VAS, visual analogue scale; BMI, body mass index.

${ }^{a}$ Boldface indicates statistical significance.

${ }^{\mathrm{b}}$ Three patients were excluded due to having both isthmic and degenerative spondylolisthesis.

and operative characteristics. The level of significance was set at $P \leq .05$.

\section{RESULTS}

Of the 58 patients with IS who underwent TLIF, $21(36.2 \%)$ were grade I and $37(63.8 \%)$ were grade II. Baseline patient characteristics are described in Table 1. The grade I cohort was younger (42.2 \pm 14.7 versus $50.6 \pm 13.1$ years, $P=.029$ ) than the grade II cohort. There was no significant difference in gender, body mass index, smoking status, level of spondylolisthesis, insurance payer, comorbidity burden, or preoperative VAS pain scores between the two cohorts $(P>.05)$.

Table 2 presents a comparison of postoperative outcomes. Arthrodesis rate (based on CT scan evaluation of the fusion mass) (Figures 1E, 1F) was lower in the grade I cohort $(85.7 \%$ versus $89.2 \%$ ), though not statistically significant. The remainder of the outcomes, including operative time, EBL, LOS, and postoperative VAS pain scores at 6 weeks, 12 weeks, and 6 months, were

Table 2. Outcomes. ${ }^{a}$

\begin{tabular}{|c|c|c|c|}
\hline & \multicolumn{2}{|c|}{ Isthmic Spondylolisthesis $^{\mathrm{b}}$} & \multirow[b]{2}{*}{$P$ Value $^{\mathrm{c}}$} \\
\hline & Grade I $(\mathrm{N}=21)$ & Grade II (N = 37) & \\
\hline Operative time, mean $\pm \mathrm{SD}$, min & $149.0 \pm 57.3$ & $149.3 \pm 36.5$ & .349 \\
\hline Estimated blood loss, $\mathrm{mL}$ & $71.9 \pm 42.8$ & $119.4 \pm 126.2$ & .143 \\
\hline Length of hospital stay, $\mathrm{h}$ & $68.7 \pm 32.0$ & $70.8 \pm 64.0$ & .666 \\
\hline \multicolumn{4}{|l|}{ Visual Analogue Scale, mean $\pm \mathrm{SD}$} \\
\hline 6-wk VAS & $4.2 \pm 2.0$ & $4.0 \pm 2.0$ & .880 \\
\hline 12-wk VAS & $3.5 \pm 1.9$ & $3.6 \pm 2.0$ & .499 \\
\hline 6-mo VAS & $3.8 \pm 2.3$ & $4.0 \pm 2.7$ & .889 \\
\hline \multicolumn{4}{|l|}{ Change in VAS, mean $\pm \mathrm{SD}^{\mathrm{d}}$} \\
\hline$\Delta$ VAS at $6-w k$ & $-2.1 \pm 2.5$ & $-3.2 \pm 2.7$ & .581 \\
\hline$\Delta$ VAS at $12-w k$ & $-3.4 \pm 3.8$ & $-4.2 \pm 2.7$ & .499 \\
\hline$\Delta \mathrm{VAS}$ at $6-\mathrm{mo}$ & $-4.1 \pm 3.4$ & $-4.1 \pm 3.1$ & .427 \\
\hline Complications, $\%(\mathrm{n})^{\mathrm{e}}$ & $0.0(0)$ & $5.4(2)$ & - \\
\hline Pseudarthrosis (CT scan) & $14.3(3)$ & $10.8(4)$ & .174 \\
\hline Arthrodesis at 1 year & $85.7(18)$ & $89.2(33)$ & .120 \\
\hline
\end{tabular}

Abbreviations: SD, standard deviation; VAS, visual analogue scale.

${ }^{a}$ Boldface indicates statistical significance.

${ }^{\mathrm{b}}$ Three patients were excluded due to having both isthmic and degenerative spondylolisthesis.

${ }^{\mathrm{c}} P$ value is from Poisson regression with robust error variance (binary outcomes) or linear regression (continuous outcomes) adjusted for age, sex, BMI, smoking status, insurance, comorbidity burden, and preoperative VAS.

${ }^{\mathrm{d}}$ Change in VAS $=$ Postoperative VAS $(6 \mathrm{wk}, 12 \mathrm{wk}$, or $6 \mathrm{mo})$-Preoperative VAS

${ }^{\mathrm{e}}$ Complications include epidural hematoma/fluid collection requiring irrigation and debridement, abdominal distension requiring nasogastric and rectal tube placement. 
similar between the grade I and grade II cohorts. There were two complications in the grade II cohort (abdominal distention requiring nasogastric and rectal tube placement, and an epidural hematoma requiring irrigation and debridement), whereas there were no complications in the grade I cohort.

\section{DISCUSSION}

The surgical management of symptomatic low grade IS in the adult population is well described in the literature and generally involves interbody fusion with instrumentation at the level of spondylolisthesis. ${ }^{13}$ Use of minimally invasive techniques has been shown to be as effective as traditional open techniques for management of single level fusions for DS. ${ }^{2}$ However, there is limited evidence examining the use of MIS-TLIF for treatment of lowgrade IS (grade I versus grade II).

As described by Scheer et al, ${ }^{2}$ MIS-TLIF with and without reduction via unilateral approach is an appropriate and effective treatment option for patients with low-grade DS. The authors observed no differences in perioperative outcomes in the reduction and no reduction cohorts; although increased EBL and arthrodesis rates at 1 year were observed in the reduction cohort.

In 2015, Kim et $\mathrm{al}^{17}$ examined the use of MISTLIF for treatment of both IS and DS. The study included a total of 41 patients, 18 and 23 of which had IS and DS, respectively. The authors noted that only 2 of the 41 patients included in the study experienced grade II spondylolisthesis. The MISTLIF procedure was performed using a single polyetheretherketone (PEEK) interbody cage with operative outcomes assessed at a mean follow-up of 1 year. The authors compared Oswestry Disability Index (ODI) and VAS scores between the 2 cohorts observing no difference in postoperative pain scores and fusion rates. However, disk height restoration improved in the IS cohort. The authors note that MIS-TLIF is an appropriate treatment option for low-grade IS and DS, however, based on the limited pathology of the patient population examined, further studies are required to examine the use of MIS-TLIF for grade II IS and DS. ${ }^{17}$

With limited data available on MIS techniques for grade II IS or higher, the present study aimed to examine the use of MIS-TLIF for treatment of grade I versus grade II IS. To the best of our knowledge, this investigation examines the largest population of patients with grade II IS undergoing a bilateral approach. The results suggest that MISTLIF is an effective surgical procedure for treatment of both grade I and grade II IS. Despite the grade I IS cohort being younger and having a lesssevere diagnosis, the grade II IS cohort experienced similar improvements in clinical outcomes when treated with MIS-TLIF. The grade I IS cohort being younger intuitively makes sense as increasing chronicity of disease may result in progression from low to higher grade spondylolisthesis. Although IS occurs in $5 \%$ to $8 \%$ of the general population, progression during young adulthood is rare due to the patient's intact resilient intervertebral discs. ${ }^{11} \mathrm{It}$ is not until the fourth and fifth decades that degenerative changes in the intervertebral discs allow progression of the anterior slippage to highgrade IS. ${ }^{7-11,13}$

The grade II IS cohort experienced increased arthrodesis rates compared with the grade I IS cohort, although not statistically significant. This finding may be due to the use of bilateral interbody cages in the grade II IS cohort. The senior surgeon elected to use bilateral interbody cages in the grade II cohort in order to increase stability as well as symmetrically open the disc space. Interbody cages rely on several factors including the osteogenetic properties and surface area of bone-to-bone contact, and distribution of stress to provide the ideal environment for fusion of the vertebral bodies. ${ }^{18}$ Although limiting the area available for graft placement, utilizing multiple interbody cages increases the surface area of bone-to-bone contact, volume of allograft or autologous bone graft material contained within the interbody cages, as well as provides a greater platform for stress distribution to aid in stability and decrease the local kyphosis. ${ }^{18-20}$ With evidence suggesting bone formation and maturation beginning at the innermost region of the intervertebral disc space close to the vertebral bodies, followed by growth extension to the outermost regions, maximizing the area in contact with the intervertebral bodies provides the most favorable environment for fusion. Meticulous endplate preparation performed under direct visualization may also have contributed to the arthrodesis rates observed.

With evidence suggesting clinical effectiveness of this therapy in grades I and II IS, the senior surgeon has begun utilizing the bilateral cage technique in patients with high-grade IS and similar improvements in clinical outcomes have been observed. 
Current literature has focused on alternative techniques for treatment of high-grade IS including number of levels fused, and type of fusion or fixation performed; however, literature describing MIS approaches for treatment of high-grade IS is limited by underpowered clinical studies and case reports to suggest one method to be superior to the other. $^{8,21}$

The present study has several limitations. First, a single surgeon at a single academic institution performed all the procedures, limiting the generalizability of the study. Second, the present study examined an alternative MIS technique on 2 lowgrade IS cohorts, previously identified to benefit from MIS-TLIF. This technique has been utilized on a relatively small population of high-grade IS patients; however, the grade III or higher patient population was too small to detect significant difference in clinical outcomes between low- versus high-grade IS. Third, the authors acknowledge that with current technology grade IV and V spondylolisthesis cannot be addressed with a minimally invasive posterior approach.

The treatment of high-grade IS remains controversial; however, a minimally invasive surgical procedure for treatment of progressive spondylolisthesis is required to reduce the operative morbidity associated with open techniques. The results of this study demonstrate equivalent clinical outcomes between grade I and grade II IS patient populations when treated with MIS-TLIF. Despite the increased anterior vertebral body translation observed in grade II IS, performing a MIS-TLIF with a bilateral approach and interbody cage placement provided sufficient stabilization and fusion. Therefore, supported by early anecdotal evidence from the senior surgeon, MIS-TLIF with bilateral approach and interbody cage placement should be examined for treatment of grade III IS; however, further research is warranted to confirm the efficacy in this patient population.

\section{REFERENCES}

1. Ahn J, Tabaraee E, Singh K. Minimally invasive transforaminal lumbar interbody fusion. J Spinal Disord Tech. 2015;28(6):222-225.

2. Scheer JK, Auffinger B, Wong RH, et al. Minimally invasive transforaminal lumbar interbody fusion (TLIF) for spondylolisthesis in 282 patients: in situ arthrodesis versus reduction. World Neurosurg. 2015;84(1):108-113.

3. Elboghdady IM, Naqvi A, Jorgenson AY, et al.
Minimally invasive transforaminal lumbar interbody fusion for lumbar spondylolisthesis. Ann Transl Med. 2014;2:99.

4. Skovrlj B, Gilligan J, Cutler HS, et al. Minimally invasive procedures on the lumbar spine. World J Clin Cases. 2015;3(1):1-9.

5. Barbagallo GM, Yoder E, Dettori JR, et al. Percutaneous minimally invasive versus open spine surgery in the treatment of fractures of the thoracolumbar junction: a comparative effectiveness review. Evid Based Spine Care J. 2012;3(3):43-49.

6. Oppenheimer JH, DeCastro I, McDonnell DE. Minimally invasive spine technology and minimally invasive spine surgery: a historical review. Neurosurg Focus. 2009;27(3):E9.

7. Floman Y. Progression of lumbosacral isthmic spondylolisthesis in adults. Spine. 2000;25(3):342-347.

8. Quraishi NA, Rampersaud YR. Minimal access bilateral transforaminal lumbar interbody fusion for high-grade isthmic spondylolisthesis. Eur Spine J. 2013;22(8):1707-1713.

9. Osterman K, Schlenzka D, Poussa M, et al. Isthmic spondylolisthesis in symptomatic and asymptomatic subjects, epidemiology, and natural history with special reference to disk abnormality and mode of treatment. Clin Orthop Relat Res. 1993;(297):65-70.

10. Faldini C, Di Martino A, Perna F, et al. Changes in spino-pelvic alignment after surgical treatment of high-grade isthmic spondylolisthesis by a posterior approach: a report of 41 cases. Eur Spine J. 2014;23(suppl 6):714-719.

11. Herman MJ, Pizzutillo PD. Spondylolysis and spondylolisthesis in the child and adolescent: a new classification. Clin Orthop Relat Res. 2005;(434):46-54.

12. Wiltse LL, Winter RB. Terminology and measurement of spondylolisthesis. J Bone Joint Surg Am. 1983;65(6):768-772.

13. Bouras T, Korovessis P. Management of spondylolysis and low-grade spondylolisthesis in fine athletes. A comprehensive review. Eur J Orthop Surg Traumatol. 2015;25(suppl 1):S167-S175.

14. Boxall D, Bradford DS, Winter RB, et al. Management of severe spondylolisthesis in children and adolescents. $J$ Bone Joint Surg Am. 1979;61(4):479-495.

15. Liu XY, Wang YP, Qiu GX, et al. Meta-analysis of circumferential fusion versus posterolateral fusion in lumbar spondylolisthesis. J Spinal Disord Tech. 2014;27(8):E282-E293.

16. Kim R, Singla A, Samdani AF. Classification of spondylolisthesis. In: Wollowick AL, Sarwahi V, eds. Spondylolisthesis: Diagnosis, Non-Surgical Management, and Surgical Techniques. New York, NY: Springer; 2015:95-106.

17. Kim JY, Park JY, Kim KH, et al. Minimally invasive transforaminal lumbar interbody fusion for spondylolisthesis: comparison between isthmic and degenerative spondylolisthesis. World Neurosurg. 2015;84(5):1284-1293.

18. Soriano-Baron H, Newcomb AG, Malhotra D, et al. Biomechanics of nested transforaminal lumbar interbody cages. Neurosurgery. 2015;78(2):297-304.

19. Postigo S, Schmidt H, Rohlmann A, et al. Investigation of different cage designs and mechano-regulation algorithms in the lumbar interbody fusion process: a finite element analysis. $J$ Biomech. 2014;47(6):1514-1519.

20. Jost B, Cripton PA, Lund T, et al. Compressive strength of interbody cages in the lumbar spine: the effect of cage shape, posterior instrumentation and bone density. Eur Spine $J$. 1998;7(2):132-141.

21. Shedid D, Weil AG, Lieberman I. A novel minimally 
invasive technique for the treatment of high-grade isthmic spondylolisthesis using a posterior transsacral rod. J Spinal Disord Tech. 2014;27(2):E41-E48.

Disclosures and COI: No funds were received in support of this work. No benefits in any form have been or will be received from any commercial party related directly or indirectly to the subject of this manuscript.

Corresponding Author: Kern Singh, MD, Department of Orthopaedic Surgery, Rush Univer- sity Medical Center, 1611 W. Harrison St, Suite \#300, Chicago, IL 60612. Phone: (312) 432-2373; Fax: (708) 492-5373; Email: kern.singh@rushortho. com.

Published 30 April 2020

This manuscript is generously published free of charge by ISASS, the International Society for the Advancement of Spine Surgery. Copyright (c) 2020 ISASS. To see more or order reprints or permissions, see http://ijssurgery.com. 